\title{
A Biochemical Analysis beyond the Muscle Contraction and its Relation with Isaac Syndrome
}

\author{
Valdemir Aquino de Freitas Neto*
}

Colégio GGE, Brazil

*Corresponding author: Valdemir Aquino de Freitas Neto, Colégio GGE, Jaboatão dos Guararapes, Pernambuco, Brazil

\begin{abstract}
Isaac Syndrome is an autoimmune disease related to the involuntary contraction of skeletal muscles. The author, thus, is going to connect it with the process of muscle contraction through a biochemical study, analysing the pathway from a nervous impulse to the open of voltage-gated Potassium channels and the releasing of $\mathrm{Ca}^{2+}$ on myofibrils. This paper intends to introduce this rare disease and to induce further researches in order to find a cure.
\end{abstract}

\section{Keywords}

Isaac Syndrome, Muscle contraction, Nervous impulse, $\mathrm{Ca}^{2+}$, Voltage-gated Potassium channels

\section{Abbreviations}

$\mathrm{Na}^{+}$: Sodium Cation; $\mathrm{K}^{+}$: Potassium Cation; ATP: Adenosine Triphosphate; ADP: Adenosine Diphosphate; $P_{i}$ : Inorganic Phosphate; $\mathrm{Ca}^{2+}$ : Calcium Cation; $\mathrm{Mg}^{2+}$ : Magnesium Cation; $\mathrm{Cd}^{2+}$ : Cadmium Cation; TnC: Troponin C; TnT: Troponin T; Tnl: Troponin I; VGKC: Voltage-Gate Potassium Channels; CASPR2: Contactin-Associated Protein like 2

\section{Introduction}

The muscle contraction process starts on the $\mathrm{Na}^{+} /$ $\mathrm{K}^{+}$pump during a nervous impulse. A nervous impulse comes from the nervous system and goes to a motor neuron through the action potential. The action potential consists in wave of positive charges that induces the open of the voltage-gated $\mathrm{Na}^{+}$channels, the $\mathrm{Na}^{+}$ions, which stay in high concentration outside the cellular membrane, flow through passive diffusion from outside to inside the cell, following the concentration gradient and the depolarization of the sarcolemma, the cell membrane of muscle cells, causes the slightly positive charge inside the cell. In that way, the positive charge inside the cell induces the $\mathrm{Na}^{+}$channel to close eventually, the $\mathrm{K}^{+}$channels to open and, then, the $\mathrm{K}^{+}$ions flow from inside to outside the cell membrane, causing the repolarization of sarcolemma [1]. Through the action potential process, the impulse is able to follow the signal to a motor neuron, consuming energy since the ATPase hydrolyses the ATP into ADP $+P_{i}$, releasing energy to restore the concentration of $\mathrm{Na}^{+}$and $\mathrm{K}^{+}$against the concentration gradient by active transport. Also, in muscle cells, that action potential is called end-plate potential.

That process can go wrong if the nervous impulse activates the pump with no order of the brain and this is the main point around Isaac Syndrome or Neuromyotonia. This type of error is important to comprehend possible causes to Neuromyotonia and to start researchers in order to find out how to prevent the involuntary contraction of skeletal muscle in rare diseases.

The author explored, through a biochemical way, over 22 recent data based on researches that involve the whole process of muscle contraction; the main works are presented in the references section and they were extracted from the most known biochemical and biophysical journals in order to develop a critical and substantiate review, meeting the majority criteria on the biochemical method.

\section{Results on the Muscle Contraction Process}

When the impulse reaches the motor neuron, it opens the $\mathrm{Ca}^{2+}$ channels and the $\mathrm{Ca}^{2+}$ ions flow to inside the motor neuron cell. The entry of $\mathrm{Ca}^{2+}$ into the cell, induces the mobilization of Acetylcholine vesicles, by docking, to the cell membrane and the vesicles fusion,

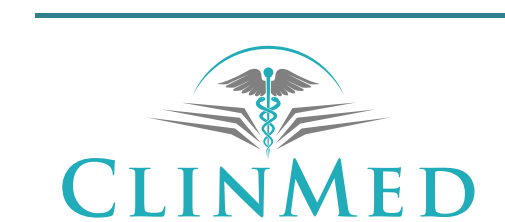

INTERNATIONAL LIBRARY 
releasing the Acetylcholine to the gap junctional in a process called Synapsis [2].

There are many receptors of Acetylcholine, called Nicotinic Acetylcholine receptors due its affinity with nicotine. In this way, when the Acetylcholine is released to muscle cells, it binds with cholinergic receptors that activate the ryanodine receptors, which are $\mathrm{Ca}^{2+}$ channels, and the $\mathrm{Ca}^{2+}$ ions are released by the sarcoplasmic reticulum, a modified endoplasmic reticulum which can afford the amount of $\mathrm{Ca}^{2+}$ ions to the muscle cell [3]. Therefore, the $\mathrm{Ca}^{2+}$ ions are released to the myofibrils. The myofibrils are a huge complex of proteins which contain the Troponin complex, Tropomyosin, Actin filaments and Myosin.

The Troponin is a protein complex with the Tropomyosin that lies between Actin filaments. The Troponin complex is made of Troponin C, Troponin T, and Troponin I [4]. The Troponin $C$ has this name because it possess 4 structural EF-Hands, which are 2 two $\alpha$-helices interrelated through a loop with negative charge ions and its configuration allows EF-Hands to bind to bivalent ions, like $\mathrm{Ca}^{2+}, \mathrm{Mg}^{2+}$ and $\mathrm{Cd}^{2+}[5]$. The Troponin I have 3 different isoforms: The slow skeletal muscle, the fast skeletal muscle and the cardiac; in general, the Troponin I works as a structural support for Troponin C, when the site $\mathrm{Tnl}-\mathrm{TnC}$ binds to $\mathrm{Ca}^{2+}$, there is a rearrangement since the 2 EF-Hands move closer, what orientates the helices, resulting in the opening of a hydrophobic path that allows the switch peptide of Troponin I to act, and, consequently, the Troponin complex is able to interact with Actin and Tropomyosin [6]. The Troponin T develops a hard-structural role because the $C$ terminal domain makes the fixation of the Troponin complex to the Actin filament, while the $\mathrm{N}$ terminal and the linking terminal stabilizes the interaction with Tropomyosin [7] (Figure 1).

The Tropomyosin normally blocks the Myosin II, the most known Myosin, binding sites in order to prevent a muscle contraction without a nerve input and without wasting ATP with no purpose [8,9]. The Tropomyosin is associated with those 3 Troponin parts (TnC, TnT, and Tnl) [10]. In a high concentration of $\mathrm{Ca}^{2+}$, the $\mathrm{N}$ terminal [11] Actinsites of TnT, called Leiomodin [12], which con-

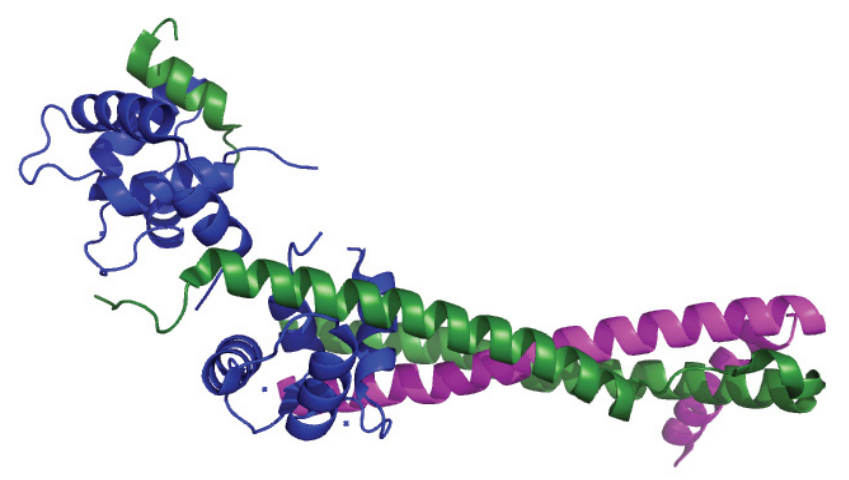

Figure 1: Structure of Troponin. tains a Tropomyosin-binding site and an Actin-capping site, when the Tnl-TnC rearrangement occurs, shifts the Tropomyosin from the closed state to the open state (azimuthal positions) and, at this state, the Tropomyosin uncovers the Actin-Myosin binding site and, also, support it; because of that behaviour, some scientists call the Tropomyosin an allosteric switch. There are a few diseases related to mutations in the $\beta$-Tropomyosin gene, called TPM2, and the most severe affect the heart contractions, like Hypertrophic Cardiomyopathy; a good way of treatment is the use of beta-blockers as medication that inhibit the abnormal activity of the Tropomyosin in heart.

The Myosin is a motor protein, what means that it plays a binding role with Actin when an input of ATP is hydrolyzed. The Myosin has several parts as Troponin and it is, basically, divided in head, neck and tail. The Myosin tail has a carboxyl-terminal group $(-\mathrm{COOH})$ in order to make the dimerization of heavy amino acids residues because of its $\alpha$-helicoidal coiled-coil form and to practice the cargo binding [13], that is, to carry through cell transportation amino acids capable of binding along the Actin filament [14]. The Myosin neck is responsible for the light chains, which belongs to the EF-Hands family and the most important is the Calmodulin, because the association of Myosin light chains is fundamental for the congregation of macromolecular complexes that work as motor protein, like dynein and kinesin. The Myosin head has an amine-terminal group $\left(-\mathrm{NH}_{2}\right)$ and it is responsible for binding to the Actin-Myosin sites, which lies on Actin filaments, via $\mathrm{N}$ terminal [15]. The hydrolysis of ATP in Myosin head occurs when there is an increase of $\mathrm{Ca}^{2+}$ by the sarcoplasmic reticulum and the Calmodulin is able to phosphorylate Myosin light chains at the serine and threonine residues [16]. Thus, the repetition of that phosphorylation promotes the activity of ATPase, hydrolysing ATP into ADP $+P_{i}$ and, consequently, releasing enough amount of energy to the contraction of Myosin head toward the Actin-Myosin binding site. When the Myosin head reaches the Actin-Myosin binding site, if and only if the Tropomyosin is on the open state, a cross-bridge is formed; so, the Myosin head releases the $\mathrm{P}_{\mathrm{i}}$, causing a conformational change in the Myosin structure that increases the affinity between Actin and Myosin (this process is called power stroke).

In that scope, there are hundreds of Myosin heads interacting with only one Actin filament, so they move it relatively fast, causing the muscle contraction by the repetition of the cycle.

\section{Discussion}

That all said, the Isaac Syndrome, which is called Neuromyotonia, is a hyperexcitability of motor nerves that causes overly muscular activity. In fact, motor nerves are different from motor neuron because the second one communicates directly with the muscular cells 
while the first one sends signals from the Central Nervous System to the Peripherical Nervous System. There are 3 main types of Neuromyotonia: Acquired, related to substances that stimulates the hyperactivity of muscles, Hereditary, related to the genetic information, and Paraneoplastic, related to the production of chemical signals as a consequence of the spread of tumour cells. By the way, the most common type of Neuromyotonia is the Acquired (around $78 \%$ of the cases), so the main focus of this manuscript is on that. Thereby, the Acquired Neuromyotonia is characterized by an autoimmune disease that induces the own body to produce antibodies which promotes the open of voltage-gate potassium channels (VGKC) [17]. These antibodies can damage cell adhesion proteins called Neurexins, which induce the excitatory or inhibitory synapses, and the most known is the Contactin-associated protein-like 2 (CASPR2) [18]. The CASPR2 is strongly coded in human cortex, precisely in the basal ganglia, by the CNTNAP2 gene code and mutations in the codification imply errors on the interaction between CASPR2 and the Contactin-2 [19] that is crucial to close the $\mathrm{K}^{+}$channels during the repolarization because of their affinity with $\mathrm{K}_{\mathrm{v}} 1$ channels (voltage-gated Potassium channel). In that way, due the error in the codification of CNTNAP2, the antibodies, the most common are the $\operatorname{lgG}_{4}$, treat CASPR2 [20] as an antigen, a foreign substance, and they prevent the binding to Contactin-2; consequently, they are not able to inhibit some nervous impulses that activate $\mathrm{K}_{\mathrm{v}} 1$ channels, allowing the impulse to reach a motor neuron and the muscle contraction occurs unduly. Beyond that, mutations in the codification of CNTNAP2 can cause inflammation of the limbic system called Limbic Encephalitis through the production of antibodies of the Leucine-rich glioma-inactivated protein 1 (LGI-1) and, because of that, some patients have high concentration of these antibodies in the blood, likely a sort of cancer [21].

Thus, the Isaac Syndrome is a continuous and, sometimes, involuntary contraction of muscles caused by a hyperexcitability of motor neurons. The symptoms include muscular stiffness and weakness due the constant activity of muscles; delayed relaxation; excessive sweating because the muscle activity increases the region temperature; tiredness and fatigue because muscle contraction consumes ATP, the muscle produces lactic acid in the absence of sufficient oxygen; beyond continuously myokymia and fasciculation. The diagnosis consists in analysis on blood and urine, electromyography, and computerized tomography scan. There is no absolute cure for Isaac Syndrome and the treatment consists in medication for muscular stiffness and pain, like anticonvulsants, and, in some cases, prednisone, that decreases immune system activity [22].

\section{Conclusion}

Therefore, is fundamental to relate biochemically from the flow of a nervous impulse to the muscle con- traction process in order to obtain a high understanding around the Neuromyotonia and to lead more researches to find out ways to prevent this disease. Nevertheless, the findings imply that the Isaac Syndrome is an autoimmune disease that affects not only the muscular system, but also the nervous system. Those studies show an intrinsic relation between errors on the codification of CNTNAP2 gene, the activation of VGKC, and, consequently, allowing the process of muscle contraction. Thus, even the Isaac Syndrome is not well studied around the scientist, this rare disease has several disadvantages for the patients and it deserves a lot of further researches in order to develop new treatments and a cure.

\section{References}

1. Forrest Michael D (2014) The sodium-potassium pump is an information processing element in brain computation. Frontiers in Physiology 5: 472.

2. Yves Dunant (1986) On the mechanism of acetylcholine release. Progress in Neurobiology 26: 55-92.

3. Shen J, Yakel J (2009) Nicotinic acetylcholine receptor-mediated calcium signaling in the nervous system. Acta Pharmacol Sin 30: 673-680.

4. Marston S, Zamora JE (2020) Troponin structure and function: A view of recent progress. J Muscle Res Cell Motil 41: 71-89.

5. Schwaller B (2010) Cytosolic Ca2+ Buffers. Cold Spring Harbor Perspectives in Biology 2.

6. Gifford Jessica L, Walsh Michael P, Vogel Hans J (2007) Structures and metal-ion-binding properties of the Ca2+-binding helix-loop-helix EF-hand motifs. Biochem J 405: 199-221.

7. Smith DA, MA Geeves (2003) Cooperative regulation of myosin-actin interactions by a continuous flexible chain II: Actin-tropomyosin-troponin and regulation by calcium. Biophys J 84: 3168-3180.

8. Bailey K (1948) Tropomyosin: A new asymmetric protein component of the muscle fibril. Biochem J 43: 271-279.

9. Bailey K (1946) Tropomyosin: A new asymmetric protein component of muscle. Nature 157: 368.

10. Brenda Schoffstall, Vincent A LaBarbera, Nicolas M Brunet, Belinda J Gavino, Lauren Herring, et al. (2011) Interaction between troponin and myosin enhances contractile activity of myosin in cardiac muscle. DNA Cell Biol 30: 653-659.

11. Norma J Greenfield, Lucy Kotlyanskaya, Sarah E Hitchcock DeGregori (2009) Structure of the N terminus of a non-muscle alpha-tropomyosin in complex with the C terminus: Implications for actin binding. Biochemistry 48: 1272-1283.

12. Boczkowska M, Rebowski G, Kremneva E, Lappalainen $P$, Dominguez R (2015) How Leiomodin and Tropomodulin use a common fold for different actin assembly functions. Nat Commun 6.

13. Liu J, Zheng Q, Deng Y, Cheng CS, Kallenbach NR, et al. (2006) A seven-helix coiled coil. Proc Natl Acad Sci USA 103: $15457-15462$.

14. Thirumurugan K, Sakamoto T, Hammer J, James R Sellers, Peter J Knight (2006) The cargo-binding domain regulates structure and activity of myosin 5 . Nature 442: 212-215. 
15. Massimo Reconditi, Elisabetta Brunello, Marco Linari, Pasquale Bianco, Theyencheri Narayanan, et al. (2011) Motion of myosin head domains during activation and force development in skeletal muscle. PNAS 108: 7236-7240.

16. Berg JM, Tymoczko JL, Stryer L (2002) Myosins move along actin filaments. Biochemistry. ( $5^{\text {th }}$ edn), New York: WH Freeman.

17. Paterson RW, Zandi MS, Armstrong R, Vincent A, Schott JM (2014) Clinical relevance of positive voltage-gated potassium channel (VGKC)-complex antibodies: Experience from a tertiary referral centre. J Neurol Neurosurg Psychiatry 85: 625-630.

18. Hart IK, Waters C, Vincent A, Newland C, Beeson D, et al. (1997) Autoantibodies detected to expressed K+ channels are implicated in neuromyotonia. Ann Neurol 41: 238-246.
19. Masuda Tomoyuki (2017) Contactin-2/TAG-1, active on the front line for three decades. Cell Adh Migr 11: 524-531.

20. Saint Martin Margaux, Joubert Bastien, Pellier Monnin Véronique, Pascual Olivier, Noraz Nelly (2018) Contactin-associated protein-like 2 (CASPR2), a protein of the neurexin family involved in several human diseases. Eur $\mathrm{J}$ Neurosci 48: 1906-1923.

21. Li W, Wu S, Meng Q, Xiaotian Zhang, Yang Guo, et al. (2018) Clinical characteristics and short-term prognosis of LGI1 antibody encephalitis: A retrospective case study. BMC Neurol 18: 96.

22. Maddison P (2002) Neuromyotonia. Practical Neurology 2: 225-229. 